\title{
Dos significados à autorregulação: perspectivas de estudantes com trajetórias acadêmicas de insucesso
}

\author{
From the meanings to self-regulation: students' prospects about academic failure \\ *Ana Margarida da Veiga Simão , **Lourdes Maria Bragagnolo Frison, ***Amélia Rodrigues Nonticuri \\ * Dra. em Educação, docente da Faculdade de Psicologia. Universidade de Lisboa. PT. \\ ** Dra. em Educação, docente do PPGE/FAE/UFPEL/BR \\ *** Assistente Social, Mestre em Políticas Sociais - PRAE/UFPEL/BR
}

\begin{abstract}
Resumo
A pesquisa, financiada pelo Conselho Nacional de Desenvolvimento Científico e Tecnológico, realizada em Instituições de Ensino Superior no Brasil e Portugal, teve como objetivo investigar as perspectivas dos estudantes com trajetórias acadêmicas de insucesso. Buscou-se compreender o significado das suas formações, identificando suas estratégias autorregulatórias. O corpus de pesquisa foi 15 estudantes de cada uma das IES. Até o momento obteve-se como resultados iniciais: a definição do campo, os sujeitos participantes, a organização e a adaptação dos instrumentos que serão utilizados na coleta. As etapas da pesquisa nos dois países têm sido um trabalho bastante minucioso para identificar e ajustar as diferenças e semelhanças entre as realidades dos países envolvidos. Palavras chave: estratégias autorregulatórias; identidade formativa, narrativas
\end{abstract}

\begin{abstract}
This research, funded by the National Council for Scientific and Technological, held in two Higher Education Institutions (HEIs) one in Brazil and another in Portugal, aimed to investigate the prospects of students with academic trajectories of failure. We tried to understand the meaning of its formations, identifying the self-regulatory strategies. The research corpus had 15 students from each of the involved IES. By the time it was obtained as initial results: the definition of the sample, participants subjects, the organization and the adaptation of instruments to be used in the survey. The steps of the research in both countries have been a very painstaking work to identify and adjust for differences and similarities between the realities of the countries involved.
\end{abstract}

Keywords: self-regulatory strategies; identity formation; narratives
Nas últimas décadas, fatores como fracasso escolar, repetência e evasão estão presentes na maioria dos contextos escolares, causando grande preocupação aos educadores.

Com a pesquisa financiada pelo Conselho Nacional de Desenvolvimento Científico e Tecnológico (Cnpq) investiga-se os fatores implícitos no insucesso escolar de univsersitários, sendo este, um problema que afeta instituições do Ensino Superior no Brasil e Portugal. Busca-se, nesta pesquisa, aproximar pesquisadoras de diferentes instituições e em contexto distintos, para mapear as dificuldades apresentadas pelos estudantes, com a intenção de, posteriormente, intervir em um trabalho que os estimulem a pensarem sobre que estratégias autorregulatórias poderão utilizar para aprender. A pesquisa incide sobre as perspectivas dos estudantes com trajetórias acadêmicas de insucesso, buscando compreender o significado que lhes dão e identificar as estratégias autorregulatórias que fazem parte do seu reportório de estudante.

No Brasil, a PRAE/UFPEL,por meio da Coordenadoria de Integração Estudantil, contempla, atualmente em torno de 3.000 (três mil) estudantes com os programas: Auxílio Alimentação, Transporte, Moradia, Instrumental Odontológico e Pré-Escolar. Um dos critérios para que os estudantes continuem recebendo os auxílios é que eles alcancem um percentual mínimo de aprovação de $70 \%$ nas disciplinas matriculadas durante o semestre. No entanto, o índice de reprovação representa um percentual em torno de $17 \%$, o que corresponde a 500 estudantes, em média com dificuldades. Diante dessa realidade, em 2012 criou o Núcleo Psicopedagógico de Apoio ao Discente com o objetivo de promover ações de estímulo e desenvolvimento da aprendizagem desses estudantes.

Na Universidade de Portugal, o insucesso no Ensino Superior apresenta estatística de cerca de $33 \%$ no território nacional. Almeida (2013) chama a atenção para a lógica que preside para determinar a taxa de insucesso, relaciona o $\mathrm{n}^{\circ}$ de diplomados num determinado ano com o $\mathrm{n}^{\mathrm{o}}$ de alunos inscritos pela $1^{\mathrm{a}}$ vez no $1^{\circ}$ ano que 
entraram na instituição $\mathrm{n}$ anos antes, sendo $\mathrm{n}$ o $\mathrm{n}^{\mathrm{o}}$ de anos requeridos para concluir o curso. Desta forma, Almeida (2013) aponta para a existência de situações muito díspares onde se incluem situações de insucesso no sentido acadêmico mais estrito: alunos que, por falta de preparação escolar, reprovam cadeiras ou anos curriculares, sofrem retenções, mas também o abandono ou o afastamento da instituição, as desistências e as transferências. De qualquer maneira a taxa de insucesso referida aponta para um fenômeno preocupante sendo necessário perceber o ponto de vista do estudante que nos pode revelar a sua visão do problema.

Os aportes teóricos desta pesquisa tem base em dois construtos: a Autorregulação da Aprendizagem e as Narrativas Autobiográficas.

A autorregulação da Aprendizagem envolve a preocupação com a aprendizagem real, com a apropriação dos conhecimentos necessários para a vida pessoal e profissional. Acredita-se que essa seria uma aletrnativa para os edudantes, porque a premissa que acompanha a autorregulação da aprendizagem é que o conhecimento se constrói à medida em que o sujeito é provocado a desenvolver competências, que possam regular e controlar a cognição, a motivação e o comportamento, com o intuito de alcançar objetivos, em uma atuação autônoma (Schunk, 1989; Zimmerman, 1989a, 1989b, 2000; Rosário et al., 2008). A aprendizagem autorregulada é um processo cíclico e multidimensional, no qual o estudante desempenha um papel ativo, num processo diferenciado de acordo com as situações (Zimmerman, 1998, 2000, 2008, 2013). Compreende-se que a aprendizagem regulada pelo próprio estudante resulta da interação de variáveis pessoais (conhecimentos, competências e motivações) que proporcionam ao estudante o planejamento, a organização, o controle e a avaliação dos processos adaptados, dos resultados atingidos e das variáveis contextuais, que o estimulam e lhe dão oportunidades de agir de forma intencional e estratégica (Lopes Da Silva, Veiga Simão \& Sá, 2004). Isso significa que não basta apenas promover espaços de reflexão e apreensão de conhecimento se as aprendizagens não forem sistematizadas pelo próprio sujeito. Zimmerman (2002), destaca que a autorregulação não é considera uma habilidade mental, ao contrário, ela é entendida como um processo de autodireção, pelo qual os aprendizes transformam suas habilidades mentais em competências. Entende-se que quando o estudante utiliza estratégias autorregulatórias estará promovendo suas habilidades mentais, fortalecendo a capacidade do aluno para aprender.

Atualmente há várias “descobertas animadoras sobre a natureza, origens e desenvolvimento de como os estudantes regulam seus próprios processos de aprendizagem" (Zimmerman, 2002), no entanto, são poucos os professores que conseguem efetivamente criar práticas que favoreçam o envolvimento do aluno em sua aprendizagem. A proposta da autorregulação pode neutralizar as diferenças individuais na aprendizagem, porque no momento em que o aluno entra em um processo de refletir para organizar sua aprendizagem, tendo controle e consciência sobre o que ele faz, seu rendimento passa a ser ativado. A função reguladora do ensino sobre a aprendizagem e a autorregulação da aprendizagem ativada pelo próprio sujeito, tendo presentes a atuação do professor em sala de aula e o envolvimento do aluno com a situação de ensino, permite acreditar que se possa modificar este quadro ou pelo menos tentar dirimí-lo.

O construto da autorregulação da aprendizagem ajuda a compreender melhor as dificuldades individuais na aprendizagem não só porque destaca o papel ativo do aluno, mas também porque considera o papel determinante do meio. Assim, o aluno, no seu papel de agente, deve desenvolver processos cognitivos, metacognitivos e motivacionais nas suas aprendizagens. É essencial colocar o aprendente perante o desafio de assumir efetivamente um papel ativo, motivado e esforçado ao longo da aprendizagem. Nota-se, no entanto, que uma precoce responsabilização do aluno pelos seus resultados ou uma exacerbada atribuição a competências pessoais, no sucesso ou no fracasso escolar, podem ter efeitos muito negativos nas crenças de autoeficácia e nos subsequentes estados motivacionais e afetivos. O meio pode proporcionar ao aluno métodos e ambientes de aprendizagem que lhe propicie a oportunidade para desenvolver as competências necessárias a uma participação ativa. Assim sendo, investigar se os estudantes a partir das narrativas de vida e formação conseguem autogerir seus objetivos é meta deste estudo. Para promover o sucesso acadêmico torna-se necessário compreender $\mathrm{o}$ fenômeno do insucesso, analisando as perspectivas dos agentes que nele intervêm, nomeadamente o contexto e os estudantes. Para perseguir esta compreensão busca-se perceber qual o repertório de estratégias de autorregulação da aprendizagem são destacadas pelos estudantes, nas narrativas de vida e de autoformação.

Nas Ciências Humanas e Sociais, Josso (2002); Abrahão (2004; 2006;2008), Souza (2006); Frison, Veiga Simão (2011) destacam que as narrativas autobiográficas podem servir como recurso metodológico em pesquisas qualitativas. Essas pesquisas utilizam-se de narrativas de história de vida e formação como instrumento metodológico, uma vez que elas se ajustam ao relato de trajetórias, que permitem identificar, por meio de quais mecanismos e processos os sujeitos chegam a uma dada situação, como se esforçam para administrar essa situação e, até mesmo, o que fazem para superá-las (Bertaux, 2010). A opção de trabalhar com narrativas autobiográficas ancoradas no construto da autorregulação da aprendizagem pode ser um processo que estimule o investimento da capacidade de reflexão, do controle e da tomada de decisão. Acredita-se que quando o estudante se narra, o faz reflexivamente, ressignificando suas vivências em experiências formadoras, as quais são importantes para o reconhecimento de suas capacidades cognitivas e emocionais. Narrar sua história de vida pode possibilitar ao sujeito fazer encontros com seu reservatório de imagens, as quais criam sentido e significado em suas escolhas e motivações futuras (Frison; Veiga Simão, 2011; Abrahão; Passeggi, 2012b; Abrahão; Frison, 2012c). 
A reflexividade é um componente crítico da aprendizagem autorregulada, ela permite ao estudante ter progresso em suas aprendizagens, fazendo ajustes em suas propostas e estratégias, investindo em ambiente e nos fatores sociais que lhe permita avançar em seu aprender. A necessidade dessa prática autorreflexiva pode ser maior em alguns ambientes do que em outros (Schunk; Zimmerman, 1998). A investigação autobiográfica tem se constituído em uma perspectiva própria, como forma legítima de construir conhecimento em uma investigação educativa e social (Bolivar, 2012). $\mathrm{O}$ autor refere que em um mundo globalizado, as pessoas sentem a necessidade de individualizarem-se, contam com referência próprias de identidade, onde o refúgio e si mesmo se converte em um porto seguro. Bertaux (2010) destaca que a narrativa de vida pode revelar os saberes práticos, orientando para a descrição das vivências pessoais e dos contextos nos quais elas se inscrevem, chamadas "narrativas de práticas".

\section{Método}

Como mencionado anteiromente, o alto índice de estudantes com insucesso na universidade provoca a necessidade de se pensar e criar condições de aprendizagem. Para isso, pretende-se mapear como se dá o enfrentamento do baixo rendimento acadêmico, por parte de estudantes. Problema considerado alvo de investigações de muitos educadores, principalmente da Psicologia da Educação (Stoeger \& Ziegler, 2008, 2010, 2011) e de algumas universidades.

Pretende-se que o resultado desta pesquisa permita avançar para o equacionar de uma intervenção alicerçada no construto da autorregulação da aprendizagem, utilizando narrativas autobiográficas. Pretende-se atuar, mapeando que estratégias autorregulatórias são utilizadas por estudantes que apresentam altos índices de insucesso acadêmico e compreender que estratégias são utilizadas pelos estudantes, para depois em um segundo momento, realizar uma intervenção que promova a utilização de múltiplas estratégias que possam auxiliar os estudantes a minimizarem as dificuldades enfrentadas em relação a aprendizagem e também contribuir com a Universidade para encaminhamento de novas propostas.

Este estudo levanta uma questão premente: a educação deve estar voltada para o desenvolvimento da autonomia do estudante, tornando-o capaz de planejar, executar e avaliar o próprio processo de aprendizagem? É importante dizer que estimular as habilidades e competências dos estudantes, para que tenham uma aprendizagem mais significativa, efetiva e duradoura é atribuição do professor e intenção desta pesquisa (Veiga Simão, 2006; Rosário et al., 2008).

A metodologia da pesquisa é qualitativa e busca, por meio de narrativas autobiográficas, escalas e questionário revelar o fenômeno investigado, dando voz a quem melhor o conhece. A amostragem será de tipo intencional, com 15 estudantes com notas inferiores a 5,0 (Brasil) e 10,0 (Portugal), que tenham tido insucesso escolar em várias disciplinas (mínimo de três).
Para coleta serão solicitadas aos universitários:

1. Uma narrativa autobiográfica descrita por estudantes com insucesso acadêmico com a intenção que revelem fatos, emoções e sentimentos percebido por si próprios, nas quais pontuem as estratégias que utilizam para estudar e aprender. No momento das narartivas os estudantes serão questionados a partir de uma pergunta detonadora: Quais são minhas dificuldades e potencialidades no processo de aprendizagem? Outras questões de investigação serão também pontuadas aos estudantes, entre elas: Como percebem seu insucesso acadêmico? Se percebem como estão construindo seu percurso acadêmico? Que variáveis pessoais, sociais e instrutivas são percebidas pelos estudantes, associadas ao percurso de insucesso acadêmico? De que modo os estudantes percebem se as estratégias autorregulatórias podem promover o sucesso acadêmico, revelando o entendimento que eles tem ao perceberem seu insucesso? Como investem na reconstrução das suas percepções relacionadas a busca do sucesso acadêmico?

2. A Escala de Autorregulação da Motivação na Aprendizagem (EAMA), de Paulino e Lopes da Silva (2012), construída para ser utilizada no Ensino Fundamental, foi adaptada para esta pesquisa com os estudantes do Ensino Superior. Tinha inicialmente 51 itens distribuídos em duas dimensões: uma que avalia as crenças motivacionais dos estudantes e outra referente à utilização por parte dos estudantes de estratégias de regulação motivacional. Após vários estudos dos grupos de pesquisa (Brasil e Portugal) esta escala passou a ter 30 itens e apenas uma dimensão, a das Estratégias da Autorregulação da Motivação da Aprendizagem. A escala consiste num instrumento de autorrelato que possibilita analisar e identificar o papel das crenças motivacionais no metaconhecimento das estratégias de Autorregulação da Motivação na Aprendizagem (EAMA) nos estudantes Essas estratégias estão organizadas em oito categorias, conforme Tabela 1.

Tabela 1

Escala de Autorregulação da Motivação para a Aprendizagem

\begin{tabular}{|c|c|}
\hline Estratégias de EAMA & Itens \\
\hline Regulação do Interesse Situacional & $1,16,23,29$ \\
\hline Regulação das Metas de & $2,12,15,17$ \\
\hline Resultado-Aproximação & \\
\hline $\begin{array}{l}\text { Regulação pela aplicação de } \\
\text { Autorreforços }\end{array}$ & $3,14,18$ \\
\hline $\begin{array}{l}\text { Regulação pela Estruturação do } \\
\text { Contexto }\end{array}$ & $4,10,19$ \\
\hline Regulação pelo Valor & $5,9,22,27$ \\
\hline $\begin{array}{l}\text { Regulação das Metas de } \\
\text { Resultado-Evitamento }\end{array}$ & $6,8,21,26$ \\
\hline $\begin{array}{l}\text { Regulação das Metas de } \\
\text { Aprendizagem }\end{array}$ & $7,11,25,30$ \\
\hline $\begin{array}{l}\text { Regulação pelas Crenças sobre a } \\
\text { Eficácia Pessoal }\end{array}$ & $\begin{array}{c}13,20,24,28, \\
30\end{array}$ \\
\hline
\end{tabular}

As respostas aos itens foram dadas numa escala tipo Likert de cinco pontos ( $1=$ nunca, $5=$ sempre $)$. 
3. Questionário de Estratégias de Autorregulação do Controlo do Desempenho (QEACD) desenvolvido por Veiga Simão e Lopes da Silva (2012) é um autorrelato que pretende identificar e avaliar estratégias individuais de autorregulação do controlo do desempenho. Foi construído para ser utilizada no Ensino Fundamental e foi adpatado pela pesquisados do Brasil e Portugal, para ser utilizado no Ensino Superior. Inicialmente o instrumento tinha oito categorias teóricas: estratégias de controlo da atenção, estratégias de controlo da tarefa, estratégias de controlo da gestão do tempo e do esforço, estratégias de controlo do estudo, estratégias de regulação emocional, estratégias de controlo por antecipação das consequências; estratégias de controlo dos recursos sociais e ambientais; estratégias de controlo de autoeficácia. Atualmente, o instrumento foi modificado e contém sete categorias teóricas, conforme a Tabela 2. Estas foram distribuídas aleatoriamente e as opções de resposta distribuem-se numa escala de Likert de cinco pontos: "Eu não sou nada assim", "Eu não sou sempre assim", "Eu não sou nem muito nem pouco assim", "Eu sou mais ou menos assim", "Eu sou mesmo assim".

Tabela 2.

Escala de Estratégias de Autorregulação do Controlo do Desempenho

\begin{tabular}{|c|c|}
\hline $\begin{array}{l}\text { Estratégias Autorregulação do } \\
\text { Controlo do Desempenho }\end{array}$ & Itens \\
\hline Testar & $1,14,18$ \\
\hline Pedir ajuda & $2,12,16$ \\
\hline Rever & $3,10,15$ \\
\hline Gestão do tempo & $4,8,20$ \\
\hline Gestâo da atençâo & $5,11,19$ \\
\hline $\begin{array}{l}\text { Avaliação do processo/monitorização } \\
\text { do estudo }\end{array}$ & $6,9,13$ \\
\hline Gestão emocional/da ansiedade & $7,17,21$ \\
\hline
\end{tabular}

\section{Resultados}

Até o presente momento obteve-se como resultados iniciais da pesquisa a definição do campo, os sujeitos participantes, a organização e a adaptação dos instrumentos acima citados, os quais serão utilizados para a coleta. Estes instrumentos foram adapatados para o Ensino Superior pelo grupo de pesquisadoras de Portugal, sendo que este grupo diminuiu e modificou as dimensões existentes nas escalas e no questionário. Posteriormente, estes instrumentos foram submetidos as investigadoras do grupo do Brasil, as quais fizeram adpatações na linguagem escrita de acordo com a realidade brasileira. $\mathrm{O}$ questionário também foi submetido aos dois grupos, os quais modificaram as dimensões e a linguagem de acordo com o contexto de cada país. No momento, estes instrumentos estão sendo aplicados em um projeto piloto para verificar se há algum tipo de inconsistência. Após a análise da coleta feita, os mesmos serão novamente revistos e, se necessário for, reorganizados.
A proposta da escrita da narrativa também foi pensada e organizada de forma que o estudante possa entender o que se pretende investigar, entendendo que, das narrativas serão extraídas significações pertinentes ao objeto de estudo. $\mathrm{O}$ instrumento de coleta das narrativas ficou assim definido:

Escreve uma narrativa autobiográfica mencionando: i) a forma como te vês enquanto estudante do Ensino Superior, as características pessoais e de ensino que interferem na tua aprendizagem e as estratégias que utilizas para estudar; ii) o que sentes relativamente ao teu percurso na universidade, como explicas o teu insucesso e o que estás fazendo para superar essa situação; iii) que expectativas tens em relação ao teu futuro no curso.

Este estudo faz parte da pesquisa Dos significados à autorregulação: perspetivas de estudantes com trajetórias acadêmicas de insucesso (MCTI/CNPQ/MEC/CAPES $\quad \mathrm{N}^{\mathrm{o}} 22 / 2014 \quad \mathrm{n}^{\circ}$ 472132/2014-8), que investiga, por meio de narrativas autobiográficas, as perspectivas de estudantes com trajetórias acadêmicas de insucesso. Pretende-se levantar fatos, emoções e sentimentos dos estudantes, verificando também quais estratégias eles utilizam para estudar e aprender.

Das narrativas pretende-se fazer uma análise compreensiva, buscando entender qual a representação mental e discursiva das relações e processos que compõem os fenômenos relatados por estudantes. Entende-se que as narrativas poderão fornecer indícios para compreender o fenomeno estudado e projetar-se como referência ao campo dos estudos da Autorregulação da Aprendizagem como meio para minimizar o insucesso acadêmico.

\section{Discussão}

É possível dizer que as etapas da pesquisa nos dois países tem sido um trabalho bastante minucioso e cuidadoso para buscar identificar e ajustar as diferenças e semelhanças entre as realidades dos países envolvidos, tanto em relação ao contexto acadêmico, quanto ao conceito avaliativo e a forma como cada universidade registra e organiza os dados, buscando estabelecer um padrão na seleção da amostra.

Como resultados preliminares pôde-se perceber as distintas realidades existentes entre o Brasil e Portugal na forma da linguagem escrita e nos encaminhamentos para a realização da pesquisa. Além disso, foi feito um levantamento dos alunos que se encontram na situação de insucesso acadêmico o que possibilitará a coleta de dados para mapear o que os estudantes percebem, como refletem sobre si e sobre o ambiente no qual estão inseridos, colocando em evidência o sujeito e sua história. Acredita-se que a identidade formativa possibilitará aos estudantes estabelecerem uma ligação entre aquilo que fazem e o que pretendem fazer, demarcando a necessidade de saber utilizar estratégias autorregulatórias, para a obtenção de melhores resultados nos desempenhos acadêmicos. Acredita-se que seja o retorno sobre si, prospectivando a mudança pessoal e reflexiva sobre sua formação. 


\section{Referências}

Almeida, A. N. (2013). Insucesso e abandono escolares no ensino superior: o caso da Universidade de Lisboa. Lisboa: Educa.

Abrahão, M. H. M. B. (2004). Pesquisa (auto)biográfica - tempo, memória e narrativas. In: M. H. M. B. Abrahão. (Org.). A Aventura (Auto) biográfica: teoria e empiria (pp. 201- 224) Porto Alegre: EDIPUCRS.

..(2008c). Le récit autobiographique - temps et dimensions de l'invention de soi. In : E. C. de Souza. (Org). Autobiographies, Écrits de Soi et Formation au Brésil. Collection Histoire de Vie et Formation - Dirigée par Gaston Pineau, (pp. 125-146). Paris: L'Harmattan.

(2012a). Pesquisar com professores na escola: contribuição da pesquisa dialógica para o desenvolvimento de aprendizagens autorreguladas. In:, A. M. Veiga Simão, L. M. Frison \& M. H. M. B. Abrahão (Orgs.) Autorregulação da aprendizagem e narrativas autobiográficas: epistemologia e práticas. Coleção: Pesquisa (Auto)biográfica $\infty$ Educação, 10. (pp. 113-154). EDUFRN, EDIPUCRS, EDUNEB, Natal, Porto Alegre, Salvador.

Abrahão, M.H.M.B. \& Frison, L. M. B.(2012c). Narrativas de autoformação: escritas de si, autorregulação da aprendizagem e reflexividade na prática pedagógica. In: M. H. M. B. Abrahão (Org.). Pesquisa (Auto )Biográfica em rede. (pp. 141-163). Natal: EDUFERN; Salvador: EDUNEB, Porto Alegre: EDIPUCRS.

Abrahão, M.H.M.B. \& Passeggi, M. C. (2012b). As narrativas de formação, a Teoria do Professor Reflexivo e a autorregulação da aprendizagem: uma possível aproximação. In: A. M. Veiga Simão, L. M. Frison \& M. H. M. B. Abrahão (Orgs.) Autorregulação da aprendizagem e narrativas autobiográficas: epistemologia e práticas. Coleção: Pesquisa (Auto) biográfica $\infty$ Educação, 10, (pp. 53-71). Natal: EDUFRN, Salvador: EDUNEB, Porto Alegre: EDIPUCRS.

Abrahão, M. H. M. B. \& Souza, E.C. (Orgs.). (2006). Tempos, narrativas e ficções: a invenção de si. Porto Alegre: EDIPUCRS; Salvador: EDUNEB.

Bertaux, Daniel. (2010). Narrativa de vida. A pesquisa e seus métodos. São Paulo: PAULUS; Natal, RN: EDUFRN.

Bolívar, Antonio. (2012). Dimensiones epistemológicas y metodológicas de la investigación (auto)biográfica. In.: M. H. M. B. Abrahão \& M. da C. Passeggi (Orgs.). Dimensões epistemológicas e metodológicas da pesquisa (auto)biográfica. TOMO I, Coleção: Pesquisa (Auto)Biográfica: temas Transversais. (pp. 27-69). Natal: EDUFRN, Salvador: EDUNEB, PortoAlegre: EDIPUCRS.

Frison, L. M. B. \& Veiga Simão. A. M. (2011). Abordagem (auto)biográfica - narrativas de formação e de autorregulação da aprendizagem reveladas em portfólios reflexivos. Porto Alegre: Educação.

Josso, Marie-Christine (2002). Experiências de Vida e Formação. Lisboa: Educa.
Lopes da Silva, A., Duarte, A., SÁ, I. \& Veiga Simão, A. M. (2004). A aprendizagem autorregulada pelo estudante: perspectivas psicológicas e educacionais. Porto: Porto Editora.

Rosário, P., Veiga- Simão, A. M., Chaleta, E. \& Grácio, L. (2008). Auto-regular o aprender que espreita nas salas de aula. In. M. H. M. B. Abrahão. Professores e alunos: aprendizagens significativas em comunidades de prática educativa. (pp. 115-132). Porto Alegre: PUCRS.

Souza, E. C. \& Abrahão, M. H .M. B. (Orgs.) (2006). Tempos, narrativas e ficções: a invenção de si. Porto Alegre: EDIPUCRS.

Schunk, D. H. (1989) Self efficacity and academic motivation. Educational Psychologist, $26(3,4)$.

Stoeger, H., \& Ziegler, A. (2008). Evaluation of a classroom based training to improve self-regulation in time management tasks during homework activities with fourth graders, Metacognition and Learning, 3, p. 207-230.

Stoeger, H., \& Ziegler, A. (2010). Do pupils with different cognitive abilities benefit similarly from a self-regulated learning training program? Gifted Education International, 26. (pp. 110.123).

Stoeger, H., \& Ziegler, A. (2011). Self-regulatory Training through Elementary-School Students' Homework Completion. In B. Zimmerman \& D., Schunk, (Eds.) Handbook of Self-Regulation of Learning and Performance. New York, NY: Routledge.

Veiga Simão, A. M. (2006). Autorregulação da aprendizagem: um desafio para a formação de professores. In: Bizarro \& Braga (Orgs.), Formação de professores de Línguas Estrangeiras: Reflexões, Estudos e Experiências (pp.192-206). Porto: Porto Editora.

Veiga Simão, A. M., Frison, L. M., \& Abrahão, M. H. (Orgs.). (2012). Autorregulação da aprendizagem $e$ narrativas autobiográficas: epistemologia e práticas. Coleção: Pesquisa (Auto)biográfica $\infty$ Educação, 10, Natal: EDUFRN, Salvador: EDUNEB, Porto Alegre: EDIPUCRS.

Veiga Simão, A. M.; Lopes Da Silva, A. (2012). A Construção de um Instrumento de Estratégias Volitivas pelo Método Q-SORT. In P. Lyra (Org) Conhecimento em Processo. Ensaios Interdisciplinares sobre Linguagem e Cognição (pp. 221-242). , Rio de Janeiro: Ed. Tempo Brasileiro EDUENF

Zimmerman, B. J. (1989a). Models of self-regulated learning and academic achievement. In, B.J Zimmerman \&, D.H. Schunk (Eds), Self-Regulated Learning and Academic Achievment. Theory, research and Practice. Progress in Cognitive Development Research. (pp.1-26). New York: Springer-Verlag.

Zimmerman, B. J. (1989b). A social cognitive view of self-regulated academic learning. Journal of Educational Psychology, 81 (3), (pp.329-339).

Zimmerman, B. J. (1998). Academic studying and the development of personal skill: A self-regulatory perspective. Educational Psychologist. 33. 
Zimmerman, B. J. (2000). Attaining self-regulation: A social cognitive perspective. In M. Boekaerts, P. Pintrich \& M. Zeidner (Eds.), Handbook of Self-Regulation. (pp. 13-39). New York: Academic Press.

Zimmerman, B. (2002). Becoming a Self-regulated Learner: an overview. Theory into practice, v.41, n.2.
Zimmerman, B. J. (2008). Investigating self-regulation and motivation: historical background, methodological developments, and future prospects. American Educational Research Journal, 45(1).

Zimmerman, B. (2013). From cognitive modeling to self-regulation: a social cognitive carrer path. Educational psychologist, v. 48. n. 3. (pp.135-147). 